\title{
Jóvenes migrantes al habla: trayectorias educativas y vidas con género
}

\section{Young Migrants Speaking: Educational Trajectories and Gendered Lives}

\author{
Anna Tarrés Vallespí
}

\section{Resumen}

El artículo muestra la tensión biográfica y el proceso educativo de dos jóvenes menores que viajaron de África a Europa a través de construcciones biográficas marcadas por el paso de sociedad de origen a sociedad de destino. El relato y la representación gráfica de la trayectoria vivida aportan elementos de reflexión sobre el encauzamiento del alumnado de incorporación tardía en nuestra sociedad. Ambas biografías se extraen de una investigación que tiene por objeto conocer qué hace posible la progresión educativa exitosa del alumnado de incorporación tardía que vive en contextos vulnerables.

A través de trayectorias encauzadas por el género y una clase social de origen media, Badra y Dakar relatan su transitar y sentir en su nuevo contexto social de destino. Un contexto que empuja a ambos a una reubicación en términos de clase y, para Badra, también en términos de género.

\section{Palabras claves}

Alumnado de incorporación tardía, trayectoria, educación, construcción biográfica, género, biograficidad.

\section{Abstract}

The article shows the biographical tension and the educational process of two minors who traveled from Africa to Europe through biographical constructions marked by the passage from the society of origin to the destination society. The story and the graphic representation of the lived trajectory provide elements of reflection on the channeling of newly arrived migrant students in our society. Both biographies are extracted from an investigation that aims to know about the successful educational progression of the newly arrived migrant students who live in vulnerable contexts.

Through trajectories channeled by gender and a middle social class in origins, Badra and Dakar relate their traveling and their feeling in the destination society. A new context that pushes both to a relocation in terms of class and, for Badra, also in terms of gender.

\section{Keywords}

Newly arrived migrant students, trajectory, education, biographical construction, gender, biographicity. 
«el Otro siempre es, en cierto modo, una parte del yo proyectada, rechazada o renegada. El Otro es el yo más la representación de la diferencia entre ambos». Samir Naïr (2010).

«¿Por qué no se concede a la gente el derecho de hablar con su propia voz?». (Gergen y Gergen, 2011)

\section{Introducción}

En el devenir entre el espacio social, y la ubicación en el espacio temporal, histórica y generacional se ubican personas y sociedades. Una matriz producto de la conjunción de la división del capital económico, cultural y social, en posiciones y posicionamientos resultantes de estructuras de poder y contradicciones estructurales y percibidas (Mannheim, 1964; Bourdieu, 1987).

En la base de dichos procesos de estratificación, la división social del trabajo asigna posiciones diferenciales a mujeres y hombres, que no se ubican igualmente en la esfera de la producción y de la reproducción, ni en el espacio público y o el privado. El género, como mecanismo cultural que nos construye binariamente como mujeres y como hombres, se expresa a la vez en las condiciones del espacio económico y social geopolíticamente delimitado y en las expectativas, las normas, los valores, o las prescripciones de los roles que lo habitan. Habitus y ordenamientos institucionales descansan en una trama de acciones cotidianas y horizontes de vida y, en esa trama, los entornos de acción (Dausien, 2007 en referencia a Alheit (1994: 179 ss.)) concretos son también 'entornos de género'.

La acumulación de vivencias biográficamente narradas muestra el transitar, el actuar y el ser entre cauces sociales. Este artículo se enmarca en el interés, siguiendo a Xavier Bonal et al., (2015), por conocer los mecanismos y agentes que contribuyen al cómo de la identificación con el proceso educativo, siguiendo a Hernández, F. y Villar, A. (2015) a través de la biograficidad presente en la narración.

La autora quiere mostrar su agradecimiento al Dr. Xavier Bonal y al Dr. Miquel Essomba, a los miembros del Grupo de Investigación ERDISC de la Universidad Autónoma de Barcelona, y a todos los jóvenes participantes, así como el impulso inicial de la Secretaría de Igualdad, Migraciones y Ciudadanía de la Generalitat de Cataluña y la colaboración de la Dirección general de Atención a la Infancia y la Adolescencia y a los centros educativos contactados.

\section{Historias de vida y biograficidad}

A través del método biográfico, se quiere conocer la vivencia del estudiar en posiciones de partida desfavorables y hacer visible en primera persona el proceso vivido a través del proceso narrado, para comprender mejor los elementos de sentido entre mundos culturales diversos en el periplo vital de la juventud. Autores como Gabriele Rosenthal o Wolfram Fischer-Rosenthal lo convierten en propuesta hacia nuestros días (ver, por ejemplo, Rosenthal (1991) y Fischer-Rosenthal (2000)). Con la conversación emergen elementos y se viven/reviven emociones que, a través del relato narrado, forman parte del modo de contarnos/representarnos a nosotros mismos. Lo social y lo personal se narran entrelazados y este lazo produce resituaciones de la propia imagen y concreciones de la vida vivida a través del sentido que imprimimos a las experiencias contadas a través de un flujo de sensaciones, anécdotas, deseos u opiniones.

El método biográfico aporta una perspectiva que, a través de las historias de vida, brinda una narración extensa de una experiencia de vida, y como exponen Bolívar y Domingo (2006) citados en DelgadoGarcía et al., (2018: 104) se trata de un método en el que «el sujeto pone el acento en los aspectos que encuentra más 
destacados de su pasado y su presente, que al mismo tiempo le son relevantes para describir, entender o representar la situación actuab〉. Así una historia de vida no tiene por objeto el conseguir validez acerca de un acontecimiento, sino que persigue el captar la visión del sujeto y de su contexto, para generar un autodescubrimiento que permitan una respuesta más profunda en relación con lo investigado.

Bettina Dausien (2007) argumenta como las construcciones biográficas, a través de la comprensión de los entornos de acción no sólo como premisas sino en lo que aportan de elementos de reproducción y de oportunidades de modificación sociales, son más que 'categorías de respuesta'. Las construcciones biográficas en ellas mismas producen la realidad. Con Peter Alheit, Dausien entiende que en esta relación dialéctica, acontece el 'principio generador' de la propia conexión y el potencial de construcción de realidad de lo biográfico. Este 'principio generador' es el que ha sido definido con el concepto de biograficidad.

El relato biográfico aporta aprendizaje ante todo cuando no niega la realidad contradictoria. La no negación del conflicto acerca a potenciales de acción (Alheit y Dausien) y permite procesos de transición.

\section{Diseño de investigación y elementos metodológicos}

Los casos presentados se extraen de una investigación que muestra elementos asociados al éxito educativo en trayectorias de jóvenes que han sido alumnado de incorporación tardía en contextos socialmente desfavorecidos prestando especial atención al orden expresivo y a la construcción biográfica. Véase esquema de la propuesta analítica contenido en la imagen 1.

\section{Imagen 1. Modelo analítico de la investigación}

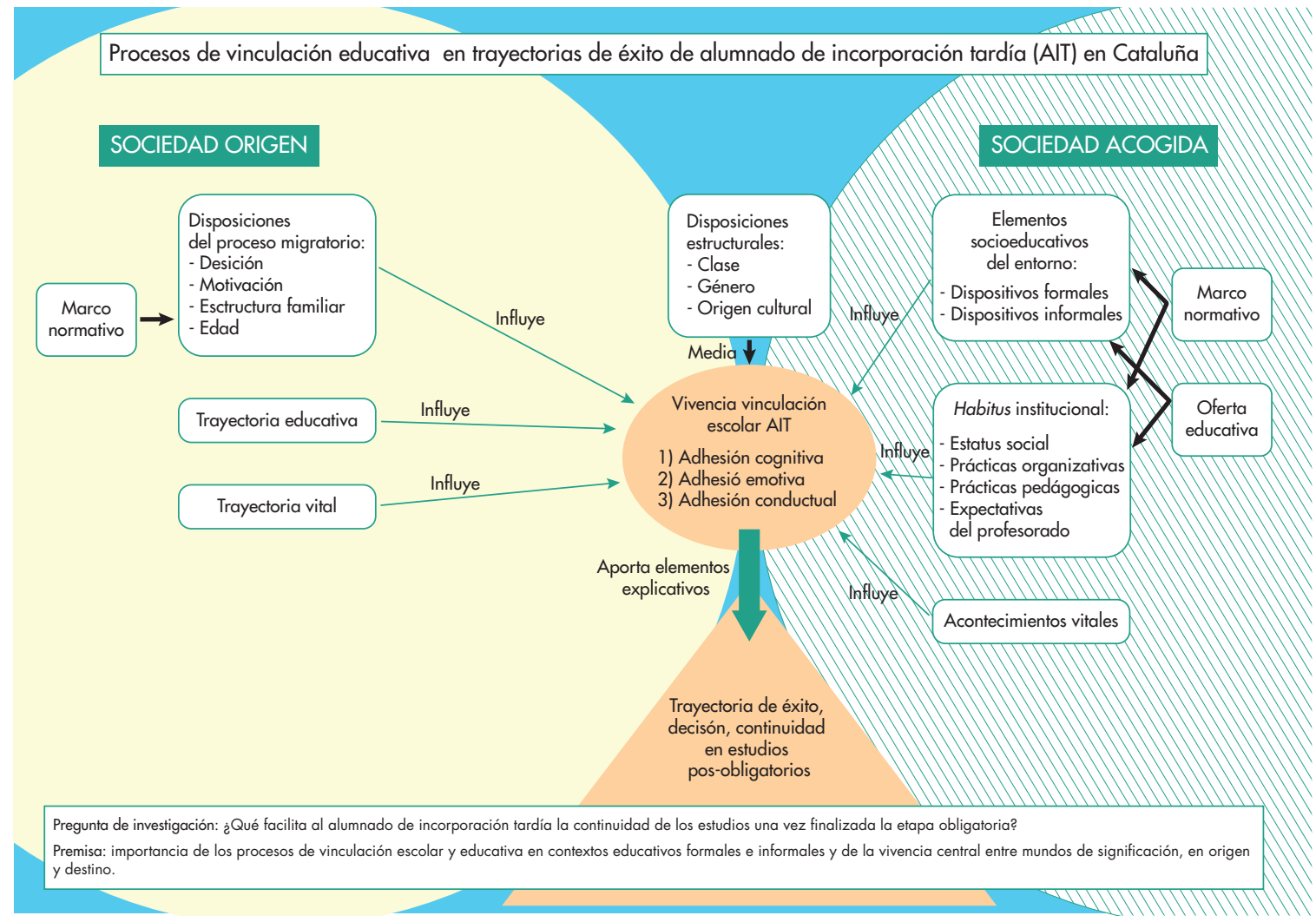

A partir de una muestra global de 30 jóvenes estudiantes de incorporación tardía al sistema educativo catalán, mayoritariamente residentes en la ciudad de Barcelona en el momento de realización del trabajo de campo, el relato y la representación gráfica de la trayectoria vital y educativa de los jóvenes son las 
herramientas que se usan para abordar la pregunta de investigación. La muestra general se divide en 2 submuestras: una de jóvenes que construyen relatos retrospectivos de su trayectoria (10 jóvenes) y otra de tipo longitudinal y formada por adolescentes (20 jóvenes) que exponen su vida año, tras año, tomando como punto de partida de la primera ola su posición de estudiantes de $4^{\circ}$ de ESO de incorporación tardía. En la Tabla 1 se aprecian las características de los componentes de la submuestra para los relatos retrospectivos.

\section{Tabla I. Muestra retrospectiva de la investigación Características de los componentes de la submuestra para los relatos retrospectivos}

\begin{tabular}{|c|c|c|c|c|c|c|}
\hline \multicolumn{7}{|c|}{ MUESTRA RESTROSPECTIVA } \\
\hline Género & Masculino & Fememino & & & & Total \\
\hline & 6 & 4 & & & & 10 \\
\hline \multirow[t]{2}{*}{ Continente origen } & Europa & Asia & África & América & Oceania & \\
\hline & 1 & 3 & 5 & 1 & 0 & 10 \\
\hline Estudios & Sin estudios & Estudios primarios & Estudios secundarios & Estudios superiores & & \\
\hline Madre & 3 & 0 & 7 & 0 & & 10 \\
\hline Padre & 1 & 3 & 5 & 1 & & 10 \\
\hline \multirow[t]{2}{*}{ Decisión emigrar } & Familiar & Personal & & & & \\
\hline & 7 & 3 & & & & 10 \\
\hline \multirow[t]{2}{*}{ Motivo emigración } & Reagrupamiento familiar & Buscar futuro mejor & & & & \\
\hline & 7 & 3 & & & & 10 \\
\hline \multirow[t]{2}{*}{ Estudios en curso } & Bachillerato & CFGM & CFGS & Grado universitario & & \\
\hline & 5 & 0 & 4 & 1 & & 10 \\
\hline
\end{tabular}

Fuente: elaboración propia.

Para ilustrar este artículo se ha trabajado con la submuestra de relatos retrospectivos que es de tipo intencional y que ha permitido seleccionar casos ricos en información, habilidades idiomáticas y habilidades comunicativas.

La metodología de la investigación se basa en una secuencia de entrevistas (ver Imagen 2) y el uso de gráficos vitales aplicados al estudio de las trayectorias educativas (Verd y Sánchez, 2010). Los gráficos presentados muestran el autoposicionamiento en la satisfacción percibida por los y las entrevistadas respecto la valoración de eventos de su trayectoria de vida y educativa. Con estas herramientas se facilita la reconstrucción de los itinerarios y trayectorias a partir del estudio de la 'vida vivida' y de las condiciones objetivas y la subjetividad de los entrevistados a través de su relato que parte de la llegada al país. En la submuestra, una vez terminadas las narrativas individuales, se ha procedido a realizar un análisis comparativo que ha permitido identificar similitudes y diferencias, temas emergentes, constataciones, etc.

\section{Imagen 2. Esquema de las etapas de aplicación de las técnicas usadas en la investigación}

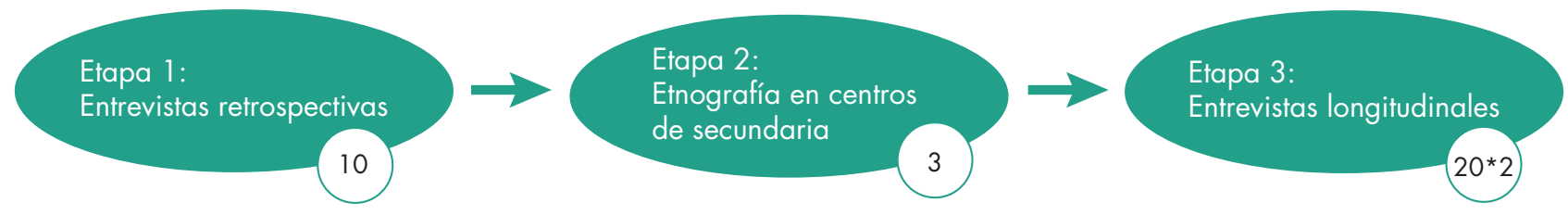




\section{Migración y momento vital, trayectorias de progreso educativo en contextos de di- ficultad}

Los jóvenes hoy ya no son una generación homogénea en términos de origen cultural y esta realidad ha transformado aulas de escuelas y centros educativos de manera permanente. Entre estos jóvenes, los más vulnerables desde un punto de vista educativo son aquellos que llegan a edad tardía; no saben hablar la lengua del país de acogida; y vienen de un país en el cual el sistema educativo es denominado por la OCDE como de baja calidad (OCDE, 2012). Es el alumnado de incorporación tardía (AIT) -los newly arrived migrante students (NAMS) - y la OCDE los define como un grupo de máximo riesgo social y hace hincapié en la necesidad que se definan políticas públicas para su apoyo específico. El estudio de los datos PISA también aporta conocimiento sobre la puntuación diferencial entre alumnado autóctono, e inmigrante de segunda o primera generación (Bonal et al., 2015; Villarroya y Bellani, 2016). Así, en los datos PISA 2012 para la competencia matemática el alumnado de origen inmigrante de primera generación -grupo donde se situaría el alumnado de incorporación tardía- éste obtiene puntuaciones 55 puntos inferiores al alumnado autóctono, mientras la segunda generación se sitúa 34 puntos por debajo ${ }^{2}$. La desigual acreditación del alumnado nativo y el alumnado de origen extranjero ${ }^{3}$ es una muestra de la desigualdad educativa de estos últimos en términos de resultados y señala la restricción en cuanto a diversidad de acceso a estudios postobligatorios del alumnado inmigrante.

La primera generación de inmigrantes no disfruta de la ventaja de conocer la cultura donde tiene que desarrollarse y los jóvenes pasan por un periodo que puede ser de intensa desorientación cuando llegan al nuevo país: «Second-generation immigrants (...) do not have to learn from scratch the cultural nuances and social etiquette that make life predictable and easier to manage. Learning the new cultural code is stressful and exhaustings (Suárez-Orozco et al., 2010: 4). Si a este elemento cultural se suma el desconocimiento de la lengua de la sociedad de acogida, el paso por el sistema educativo se hace todavía más complejo y, aún más cuando la edad de incorporación es tardía (Gjefsen y Galloway, 2013).

Pero hay jóvenes que presentan trayectorias que superan estas dificultades. En la investigación longitudinal sobre jóvenes inmigrantes en los Estados Unidos LISA ${ }^{4}$ se muestra una tipología de trayectorias que, además de trayectorias de bajo rendimiento, lento declinar, y declinar apresurado, tipifica trayectorias de alto rendimiento y 'mejoradores' (improvers). Es decir, trayectorias de jóvenes quién, a pesar de las dificultades, mejoran las calificaciones con el paso del tiempo: un 30\% de la muestra no baja el rendimiento académico y de estos, un $22 \%$ son estudiantes de alto rendimiento y un $10 \%$ mejoran progresivamente su calificación (Suárez-Orozco et al., 2010: 27). Los jóvenes 'improvers' presentan dos elementos diferenciales respecto el resto de jóvenes: llegan al país después de haber sufrido algún tipo de trauma que altera sus condiciones de vida y, todos ellos, encuentran algún mentor en el país de acogida que facilita el impulso en su trayectoria académica (Suárez-Orozco et al., 2010). En nuestro país, Aparicio y Portes (2014), hacen un seguimiento longitudinal de una muestra de jóvenes inmigrantes en Barcelona y Madrid, y subrayan la importancia para la integración de elementos propios de los diferentes colectivos estudiados, pero también de factores dependientes de la sociedad receptora como por ejemplo los segmentos sociales donde estos jóvenes se integran y el papel de los estereotipos.

\footnotetext{
2 Villaroya y Bellani 2016: 15. Estas autoras también recuerdan que diferenciales de puntuación de 40 puntos equivalen a un año de retraso estimado en la enseñanza.

3 Como muestran, por ejemplo, los últimos datos aportados en Cataluña por la Secretaría de Igualdad, Migraciones y Ciudadanía de la Generalitat en el Informe sobre la integració de les persones immigrades a Catalunya 2015 (Pinyol-Jiménez, 2016).

4 Acrónimo de The Longitudinal Immigration Student Adaptation, investigación desarrollada por el Institute for Immigration, Globalization, and Education de la UCLA a partir de datos anuales de 400 participantes a lo largo de 5 años, desde 1997.
} 
Carles Serra y Josep Maria Palaudàries indicaban el 2009 el interés en conocer las características, las estrategias y las experiencias en los centros educativos y en los contextos sociales y familiares del alumnado migrante con trayectorias de continuidad tras la educación posobligatoria (Sierra y Palaudàries, 2010a; 2010b). Y, en ese sentido, María Paz Sandín resumía que los discursos académicos hasta el momento «se han centrado, fundamentalmente, en las 'necesidades' y 'limitaciones' de los estudiantes y muy poco en el estudio de sus potencialidades». (Sandín, 2014: 11).

\section{Alumnado de incorporación tardía en estudios superiores: trayectorias con y contra la norma social. Estudio de dos construcciones biográficas contrapuestas en térmi- nos de género}

Los jóvenes entrevistados presentan trayectorias de mejoramiento de la percepción de la posición educativa, con un primer momento de gran declinar en el rendimiento educativo y una progresiva recuperación ligada en primer término al logro de la competencia comunicativa y en segunda instancia a la consolidación de sus capacidades a partir de la estabilización de las condiciones de educabilidad y de la intervención de elementos de orden expresivo que actúan como pivotes orientadores de la trayectoria.

La llegada al país de acogida es un punto de inflexión, en negativo o en positivo, para el conjunto del alumnado de incorporación tardía entrevistado. Todas las trayectorias autorepresentadas a través de los gráficos de satisfacción educativa tienen líneas de desarrollo ascendentes a pesar de presentar en casi todos los casos desarrollos abruptos.

A continuación, se muestran dos biografías de jóvenes de incorporación tardía al sistema educativo de la sociedad de acogida, que llegaron entre 2009 y 2010 a Cataluña siendo menores de edad. Un caso es el de Dakar', un chico africano que emigra por decisión propia para 'encontrar un futuro mejor' y Badia, chica marroquí que, tras casarse, llega a destino por reagrupamiento familiar.

\section{Caso 1: Dakar, la progresión en la expectativa de éxito: «allí, con 16 años no eres un niño»}

La trayectoria de Dakar, un chico originario de Guinea Conakry, está marcada por su decisión de emigrar para buscar un futuro mejor en Europa. Llega a Cataluña como menor no acompañado con 16 años y se encuentra con la sorpresa de no poder trabajar porque no dispone de los requisitos legales para la obtención del permiso de trabajo. Es tutelado por la administración pública y empieza el contacto con una red de entidades que le permiten retomar una dinámica educativa que era errática en origen. Es uno de los hijos grandes de una familia donde el padre, hombre culto y pequeño propietario de una tienda, ha tenido 3 esposas y tiene 14 hermanos. Es un caso de joven emprendedor a quién Barcelona como ciudad ha cautivado y que al no poder trabajar opta por una carrera académica gracias a una beca de estudios. Tiene en la experiencia de poder participar en un campo de juventud internacional, gracias a un primo y el apoyo de una asociación juvenil, el ocio educativo y la participación informal en 'flash mobs' la manera de empezar su apertura a la sociedad de acogida.

En el momento de la entrevista, lleva 5 años en el país.

5 Todos los nombres son ficticios. 
Gráfico 1. Trayectoria vital y educativa de Dakar

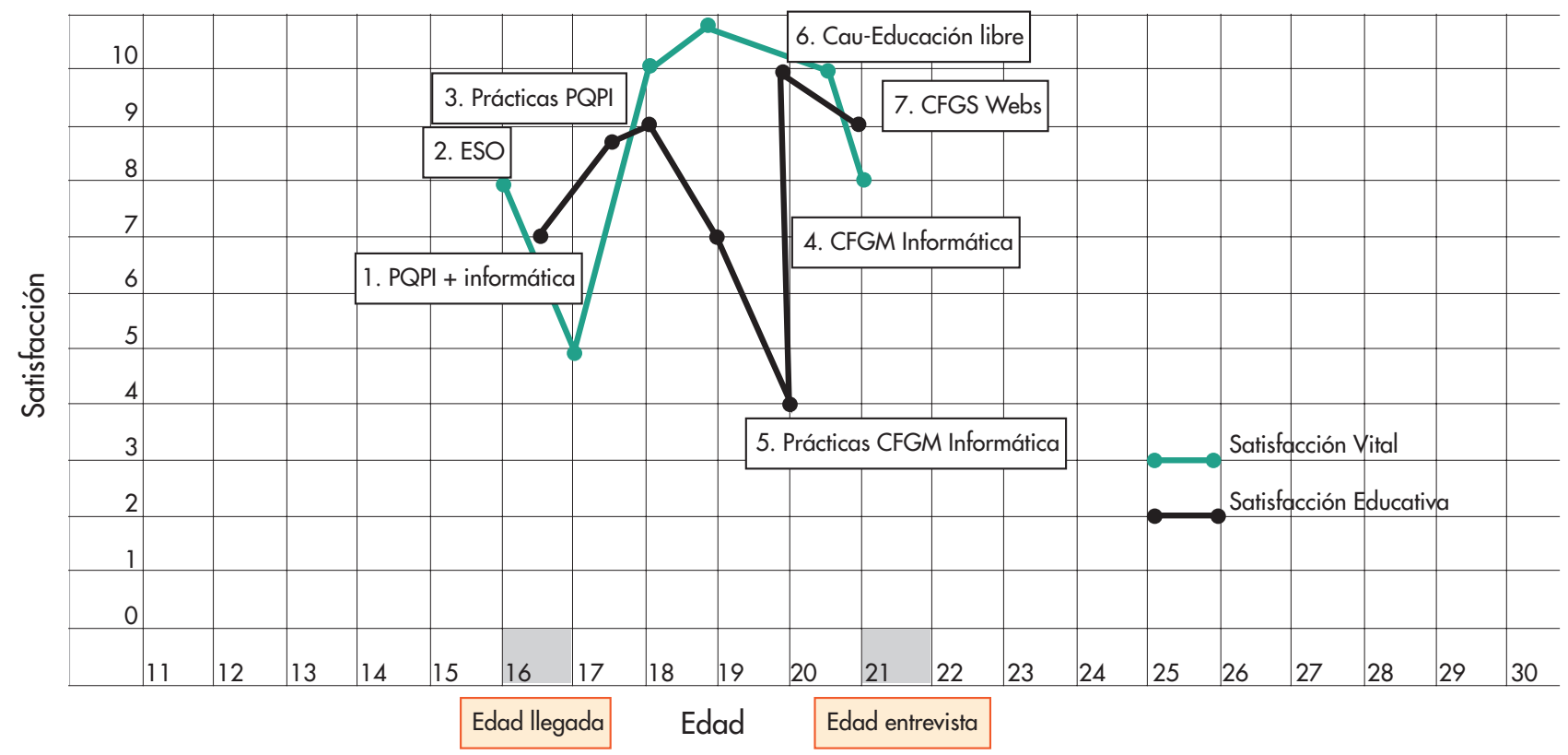

Fuente: elaboración propia a partir de datos primarios.

Como se muestra en el Gráfico 1, Dakar mantiene una trayectoria vital siempre ascendente que lo sustenta en los momentos de dificultad educativa. Esta trayectoria solo se muestra alterada por una baja valoración de los primeros momentos en la llegada a la sociedad de acogida. Este joven subraya la experiencia de llegar con un objetivo y ver que no puede cumplirse: «Realmente yo venía con la idea de venir a Europa a trabajar, no? Para encontrar una vida mejor. Pero cuando llegué encontré la situación que habia y tal y, realmente, tuve que cambiar el chip. Adaptarme a la situación. Porqué yo no habia pensado en venir aqui a estudiar. Incluso teniendo 16 años. Porqué en África, (...) alli, 16 años era adulto. Alli, con 16 años no eres un niño. Y aquí 16 años quizás sì».

Dakar en su cultura de origen es un adulto y vive como tal y en la cultura de destino es un niño; un joven que no puede vivir la vida como un adulto y al que la administración pública hasta los 18 años debe tutelar.

Es el único caso de la submuestra de relatos retrospectivossin interrupciones en la trayectoria educativa.

Dakar tiene mucha facilidad para comunicarse y a lo largo de su primer año en el país supera el obstáculo que significa la carencia de recursos comunicativos en la lengua de acogida. Aprende castellano y se defiende en catalán e inicia una progresión educativa continuada.

Antes de la migración era absentista en el sistema educativo de la sociedad de origen, momento que él mismo valora de manera muy negativa. La llegada al país de acogida significa la comprensión de los requerimientos de formación para lograr su objetivo vital de tener una vida mejor: «... el sueño que yo tenia, (...) porqué Barcelona es... ;Buf! (admiración) dentro del mundo suena como la ciudad mágica. (...) Yo vine con la idea de trabajar, de ayudar a mi familia». Pero al tener una oferta educativa, aprovecha la oportunidad: «yo, por una parte me interesaba la informática, pero nunca me habia planteado que voy a estudiary tal (...) No sé, era como empezar un camino que no conocía. (...) pero lo acabé sacando, junto con la ESO, todo en un año. Y, realmente, para mi fue un exitazo». 
En su trayectoria, la obtención de buenas calificaciones en las oportunidades educativas es el elemento fundamental para la continuidad: «...y las notas que termine sacando y todo, pues digo, jvoy a seguir.» La buena orientación del trayecto académico que tendrá que desarrollar para lograr una titulación actúa como elemento motivacional: «Veo cuando acabaré y tal. Y todo esto me motiva».

Con estas premisas, enumera experiencias extraeducativas que aportan un importante valor a su voluntad de trabajar autónomamente y lo enriquecen. Así por ejemplo Dakar destaca que: «Viaje a Francia, porqué participé en un campo de trabajo. (...) yo no sabia que tenía esa parte de creatividad... Esa parte de soltura con la gente».

\section{Caso 2: Badia, la progresión a pesar de la crisis identitaria: "estás como en un puente»}

Badia es una chica que tiene 17 años cuando llega a Cataluña desde una ciudad del Marruecos por reagrupamiento familiar, acabada de casar. Llega bajo la promesa de su esposo, único familiar en el país, de poder seguir estudios. En origen mantiene una trayectoria de alto rendimiento en los estudios, pero en destino su esposo no le permite proseguir dichos estudios. Esta situación es causa de maltratos y de divorcio en 4 meses y origina el trauma de encontrarse en un país extranjero sin apoyo familiar. Después de una orden de alejamiento y de un cambio de provincia, es acogida como menor en un centro y la situación personal se agrava todavía más al no poder estudiar por carencia de convalidaciones.

Proviene de una familia de clase mediana y profesiones liberales, ambos progenitores con estudios secundarios, pero con una visión tradicional del rol de la mujer como esposa.

\section{Gráfico 2. Trayectoria vital y educativa de Badia}

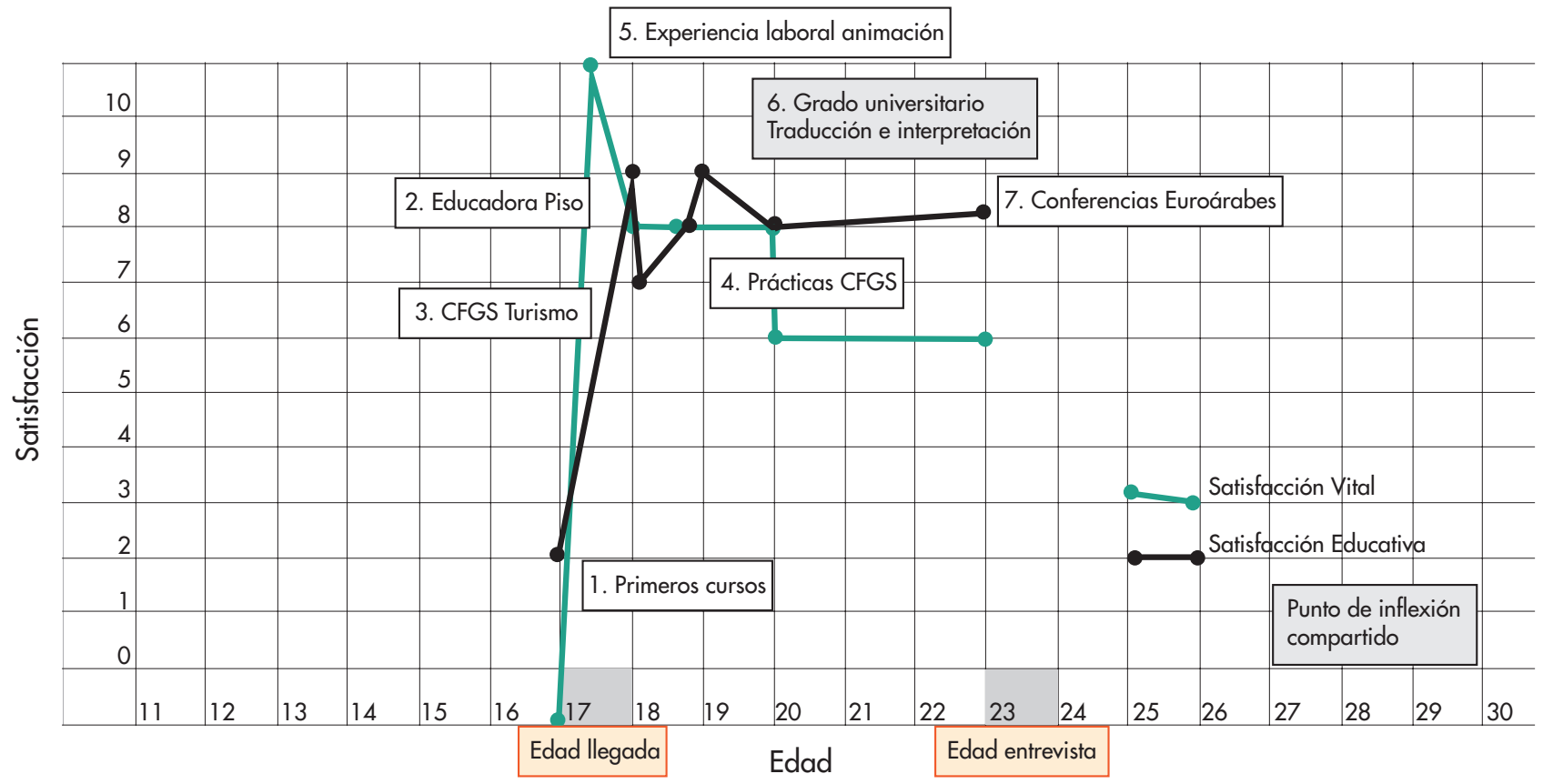

Fuente: elaboración propia a partir de datos primarios.

El origen de la trayectoria es el punto de corte del maltrato y el divorcio. En la representación gráfica (Gráfico 2) muestra su insatisfacción vital y los logros educativos que en el relato expresa como éxitos de su 'exigencia', en lucha constante con el cauce fijado para ella en su sociedad de origen y destino. 
A partir de su relato, esta joven muestra que sufre una triple ruptura: de género, de clase y de origen cultural. Un desarraigo que la sitúa en un callejón sin salida del cual consigue escapar, pero con mucho desgaste personal y viviendo situaciones de gran estrés que a menudo sitúan a la víctima en posiciones de indefensión. Particularmente duro en términos biográficos es el divorcio implica dejar de existir para su cultura y para su familia. En sus propias palabras, Badia dice: «Tenía 2 opciones: o volver, y yo sabía que con la vuelta a mi país no voy a vivir más de lo mismo: soy una chica divorciada, ...Con eso ya te lo digo todo. ¡Ser divorciada! Con eso ya se acabó mi vida. A los 3 meses o 4 ya me buscaran otro, y venga. Se acabó. Yo lo tenía tan claro que no. Que no quiero, que esto está descartado. Entonces tenía que aguantan». Con el paso del tiempo, la separación se mantiene como un estigma que no le permite mantener relaciones de aceptación ni con la familia de origen ni con personas de su cultura en Cataluña o en Marruecos: «Conocidos muchos. Amistades no tengo... Gente de mi cultura, básicamente, ni lo pienso. La verdad es que todavía no he superado eso, entonces no... No saben nada. Saben que soy una chica que ha venido aqui para estudiar. Y punto».

Su estrategia pasa por el fortalecimiento de su voluntad individual de estudio y de superación de las dificultades de cara al objetivo educativo que decide antes de la migración: «Yo no descarte en ningún momento lo de estudiar. Yo tenía claro que quería seguir estudiando. (...) Entonces, se acabó (pero) soy menor. Sin familia aqui. Era él el unico. Era mi todo en aquellos tiempos. Todo eso. Hasta que ya un día he dicho 'Prou'. Seguir así y acabo con mi vida... Porque, a ver... de una manera u otra estaba acabando con mi vida. Voy a acabar como muchas más que llegan aquí y ya está. Se conforman con lo que hay. Hacer pan y tener hijos. Es lo que hay».

Es un caso de desarraigo múltiple, como mujer y dentro de una clase mediana, pero también en términos culturales: "Yo vengo de una cultura que... donde hay más calor humano, sinceramente. Mucho más. La gente, aparte de eso, el ritmo no está tan acelerado como aquí. Eso ayuda. (...) Somos muy de abrazar y eso. Y llegas aquíy... iNo bay eso! (...) Somos muy de familia y de comer y jajaja. jijiji y aqui no. Aqui cada uno tira por su lado».

Pasa por un centro de acogida para mujeres maltratadas y por centros de menores hasta que tiene 18 años y puede ir a vivir a un piso de acogida. Pero allí, topa con otros obstáculos burocráticos puesto que un requisito es estar estudiando y ella todavía está pendiente de la convalidación de su titulación. Dice: «Son pisos con las mismas normas, siguiendo las mismas normas,... por eso muchos jóvenes dicen no. Ya está. No quiero más normas. Claro, yo, la verdad, con mi situación lo acepté, estaba en un piso (...) y uno de los requisitos es estar estudiando. ¡Y no tenía ni el título convalidado! ¡Tenía el resguardo del trámite de la titulación!» Esta situación se resuelve a través del trabajo conjunto de una educadora y una directora de centro que le facilitan el acceso a los estudios cuando la situación legal todavía no está suficientemente documentada.

Verbaliza la distancia cultural y la cosificación que sufre al sentirse a menudo 'un numerito' entre muchas personas que se cuidan de ella sin establecimiento de vínculo afectivo o humano: «Aguantar a toda esta gente... (...) Eres un caso más. Un numerito... Yo lo llamo un expediente más. Tú vienes y lloras, ataques de ansiedad, cosas. Te calmas. Te vas. Él también, le puede afectar más o menos. Se va. Se acabó la jornada laboral. Se va. Alli te quedas. (silencio) Acabas con este ritmo. (...) Tienes 4 tutores por la mañana y 4 por la tarde y venga a contar la misma historia, 8 veces. Entonces, no. Cansa. Tener una persona siempre, que sabe de qué va la misma cosa, la verdad que ayuda mucho. (Respira-suspira) Todo esto, yo, lo he tenido al mismo tiempo que mis estudios》. Es excepción una mujer adulta que se convierte en referente y mentora al establecer una relación percibida como sincera, fuera de los vínculos de la profesionalidad, de manera gratuita: «En toda mi trayectoria, vamos a llamarla así, en pisos, educadores, esta señora es importante. Y no viene de nada. Por tema... Vamos a decir religioso, era monja. Entonces, quieras o no... De no tratarte como un número... iEsto es mucho! Porque el resultado 
que da esta señora no lo da ningún colectivo. Aunque sea de 10 educadores. No lo da. (...) Ella tampoco tenía mucho tiempo (...) Necesitaba ese apoyo moral, de una persona un poco más cercana. Que no esté alli fichando para bablar conmigo y fichando para acaban».

Es en el momento de restablecimiento del punto de partida premigratorio por su reubicación en el nivel académico prefijado en el momento de la salida de la sociedad de origen, cuando consigue reestablecer una trayectoria de estudio y mantener algunos vínculos afectivos de calidad cuando se vuelve a reubicar como persona y progresa en la trayectoria educativa: «Soy Badia. Otra vez, soy yo».

\section{Conclusión:}

\section{Biograficidad y género en trayectorias educativas de jóvenes de incorporación} tardía a la sociedad de destino

Hombres y mujeres no viven ni narran su vida de igual manera; más allá de su ser individual, se constituyen en cauces materiales y simbólicos distintos. El artículo ha mostrado trazos biográficos de 'presuntos ganadores' en términos de éxito en sus trayectorias educativas: son jóvenes que ingresan en estudios en la sociedad de destino de modo tardío y, cuando todos los indicadores les situaban en la máxima desventaja (tanto en acceso, como en procesos, como en resultados), ellos han logrado proseguir su trayectoria educativa hacia los estudios superiores. Además, su biografía aporta, entre distintos grados de pérdidas y quiebras, narrativas alienadas con su representación de género. El ser masculino hegemónico (Connell, 2005) permite fluir normativamente dentro de lo que Connell (2001), siguiendo a Jordan (1995), identifica como las 'warrior narratives' y actuar como tales: chicos que viven una migración, pero esta no significa rotura alguna en su socialización de género. Pero al ser femenino tal devenir le es vedado. Para ellas la narratividad y el cauce social es otro y Caperucita vivirá como niña y lobo, contradictoriamente. Con y contra la norma social.

En ambas biografías, salir del país de origen culturalmente como adultos y llegar al país de acogida como menores refuerza la vivencia de ruptura. Pero mientras en el caso masculino el patrón de género para reubicarse sigue intacto, en el caso femenino, no es posible mantenerse en un patrón de género que tanto en origen como en destino la encauza hacia posiciones de marginalidad en los entornos de acción (Dausien, 2007 en referencia a Alheit,1994).

Dakar parte de una socialización masculina que debe readaptar al paso de la sociedad de origen a la sociedad de destino, pero dicho transitar, su trayectoria, muestra una posible continuidad que él aprovecha en su paso por los distintos marcos institucionales y reinventa, reinventándose a si mismo: «Allí, con 16 años no eres un niño (...) Pero cuando llegué encontré la situación que había y... tuve que cambiar el chip».

Badia parte de una socialización en femenina que, en el paso de la sociedad de origen a la de destino, rompe con la sumisión a lo masculino hasta su reinvención, por cauces institucionales y de sentido posibles en la nueva sociedad que la sitúan en un reencontrarse a sí misma en unos nuevos términos: «Soy Badia. Otra vez soy yo».

El divorcio es la ruptura con su posición y su ser en el mundo y Badia muestra a través de su narración que siente que ya no pertenecer a ninguna cultura ni a ninguna sociedad; se siente «como en un puente», con un pie a cada orilla, pero en ninguna parte o «como una inquilina», permanentemente ocupando la casa de otro «que es el dueño».

Encauzada fuera de la norma, el 'ser mujer' la sitúa en una lucha de sentido constante por la pertinencia material y cultural. La biograficidad-como principio generador dialéctico en las construcciones 
biográficas- alumbra cauces vividos y narrativas sociales en los relatos y representaciones observados/ construidos.

\section{Referencias bibliográficas}

Alheit, Peter y Dausien, Bettina (2007): “La construcció biográfica de la realitat” en Francesc Jesús Hernàndez Dobón (ed.): En el curs de la vida. Educació, formació, biograficitat i gènere. Xàtiva: Edicions del CReC. Diputació de València.

Aparicio, Rosa y Portes, Alejandro (2014). Crecer en España. La integración de los hijos de inmigrantes. Barcelona: Obra Social ‘la Caixa’. Colección Estudios Sociales núm. 38.

Ayuntamiento de Barcelona (2016). Informes estadístics. La población estrangera a Barcelona. Gener 2016. Núm 64. Barcelona.

Bonal, Xavier; Castejón, Alba; Zancajo, Adrián y Castel, José Luis. (2015). Equitat i resultats educatius a Catalunya. Una mirada a partir de PIS A 2012. Barcelona: Fundació Jaume Bofill. Col lecció Informes Breus, núm. 60.

Bourdieu, Pierre (1987). La distinción. Criterios y bases sociales del gusto. Madrid: Taurus, 1998.

Connell, Robert W. (2001). Educando a los muchachos: nuevas investigaciones sobre masculinidady estrategias de género para las escuelas (en línea). http://www.redalyc.org/articulo.oa?id=105115268013, consultado el 23 de marzo de 2018.

Connell, Robert W. y Messerschmidt, James W. (2005): "Hegemonic masculinity. Rethinking the Concept”. Gender \& Society, Vol. 19 No. 6, 829-859 DOI: 10.1177/0891243205278639.

Dausien, B. (2007): "Sobre la construcció social de la biografia” en Hernàndez Dobón, F. J. (ed.) En el curs de la vida. Educació, formació, biograficitat i gènere. Xàtiva: Edicions del CReC. Diputació de València.

Dausien, Bettina (2007): “Sobre biograficitat i gènere” en Francesc Jesús Hernàndez Dobón(ed.): En el curs de la vida. Educació, formació, biograficitat i gènere. Xàtiva: Edicions del CReC. Diputació de València.

Delgado-García, Manuel; Coronel Llamas, José Manuel; Boza Carreño, Ángel (2018). “Un recorrido particular por la educación: reflexiones a partir de una historia de vida". Veus silenciades. Narratives per a la transformació educativa, Revista Educar, vol. 54 (1), 101-121. https://doi.org/10.5565/rev/educar.921

European Commission (2013). Study on Educational Support for Newly Arrived Migrant Children. Brussels: European Commission.

Fischer-Rosenthal, Wolfram (2000): "Biographical work and biographical structuring in present day societies" en Joana Bornat, Prue Chamberlayne y Tom Wengraf (eds.): The Turn to Biographical Methods in Social Science. Londres: Routledge.

Gergen, Kenneth J. y Gergen, Mary (2011). Reflexiones sobre la construcción social. Barcelona: Paidós Ibérica.

Gjefsen, Hege Marie \& Galloway, Taryn Ann (2013). Young Immigrants: Age at Migration and Performance in Education. Preliminary draft, 15 April 2013.

Hernàndez, Francesc J. y Villar, Alícia (2015) (eds.). Educación y biografías. Perspectivas pedagógicas y sociológicas actuales. Barcelona: Editorial UOC. Colección Manuales (Pedagogías Contemporáneas). 
Jordan, Ellen (1995). "Fighting Boys and Fantasy: The Construction of Masculinity in the Early Years of School”. Gender and Education, 7, 69-86.

Mannheim, Karl (1964). El Problema de una sociología del saber. Madrid: Tecnos, 1990.

Naïr, Sami (2010). La Europa mestiza. Inmigración, ciudadanía, codesarrollo. Barcelona: Galaxia Gutenberg.

OECD (2012). Untapped skills. Realising the potential of immigrant students. Paris: OECD Publishing.

Pinyol-Jiménez (coord.) (2016). Informe sobre la integració de les persones immigrades a Catalunya 2015. Departament de Treball, Afers socials i famílies, Secretaria d'Igualtat, Migracions i Ciutadania, Generalitat de Catalunya.

Rosenthal, Gabriele (1991). "La estructura y la Gestalt de las autobiografías y sus consecuencias metodológicas”. Historia y Fuente Oral núm. 5, 105-110.

Sandín, María Paz et al., (2012). Resiliència i èxit escolar en l'alumnat de secundària de procedència estrangera. Grup de Recerca en Educació Intercultural (GREDI). Universitat de Barcelona.

Serra, Carles y Palaudàries, Josep Miquel (2010a). Continuar o abandonar. L'alumnat estranger a l'educació secundària. Informes Breus, $\mathrm{n}^{\circ}$ 26. Barcelona: Mediterrània - Fundació Jaume Bofill.

Serra, Carles y Palaudàries, Josep Miquel (2010b). "Deficiencias en el seguimiento del abandono escolar y trayectorias de continuidad del alumnado de origen inmigrado". Revista de Educación, número extraordinario 2010.

Suárez-Orozco, Carola; Suárez-Orozco, Marcelo M. y Todorova, Irina (2010). Learning a New Land. Immigrant students in American Society. Cambridge, Massachusetts: Harvard University Press.

Verd, Joan Miquel y Sánchez, Nuria (2010): “La reconstrucción de la trayectoria biográfica en los gráficos vitales de satisfacción vital”. Prisma Social, núm. 4. Revista de Ciencias Sociales

Villarroya, Anna y Bellani, Daniela (2016). Indicadors sobre educació. En L'educació com a ascensor social. Barcelona: Observatori Social de 'la Caixa', Dosier núm. 1.

\section{Nota biográfica}

Anna Tarrés Vallespí estudió Periodismo y Sociología en la Universidad Autónoma de Barcelona (UAB). Ha desarrollado actividades en el ámbito de la educación en el tiempo libre y en el mundo asociativo. Sus investigaciones de investigación y organización se han realizado en su mayoría desde el tercer sector. En los últimos años se ha dedicado al Panel de Desigualdades Sociales de Cataluña, un proyecto de la Fundación Jaume Bofill para el estudio de la sociedad catalana. También ha desarrollado un proyecto de empoderamiento de jóvenes a través de las artes, las nuevas tecnologías y los media con el Equipo de Investigación en Diversidad e Inclusión en Sociedades Complejas (Departamento de Pedagogía Aplicada, UAB) y ha trabajado como consultora en la Universitat Oberta de Catalunya. 